\title{
Maximally robust state-feedback controllers for stabilization of plants with normalized right coprime factor uncertainty*
}

\author{
Pramod P. Khargonekar \\ Department of Electrical Engineering and Computer Science, \\ The University of Michigan, Ann Arbor, MI 48109-2122, USA
}

\section{Duksun Shim}

Department of Aerospace Engineering, The University of Michigan, Ann Arbor, MI 48109-2140, USA

Received 19 November 1992

Revised 13 February 1993

Abstract: It is shown that a maximally robust state-feedback controller for a plant with normalized right coprime factor uncertainty is given by the solution to a standard linear quadratic regulator problem.

Keywords: Robust stabilization; linear-quadratic regulator; state-feedback.

\section{Introduction}

It is well known that the linear quadratic statefeedback regulator enjoys remarkable robustness properties $[3,5]$. In this paper, we consider the state-feedback version of the problem of robust stabilization of a plant subject to perturbations in the normalized right coprime factors. Robust stabilization of plants with (not necessarily normalized) coprime factor uncertainty was first studied by Vidyasagar and Kimura [7]. Glover and

Correspondence to: Prof. P.P. Khargonekar, University of Michigan, Department of Electrical Engineering and Computer Science, The University of Michigan, Ann Arbor, MI 48109-2122, USA.

* Supported in part by National Science Foundation under grant no. ECS-9001371, Airforce Office of Scientific Research under contract no. AFOSR-90-0053, Army Research Office under grant no. DAAL03-90-G-0008.
McFarlane $[2,4]$ have given a complete solution to this problem in the output feedback case. This problem is also related to the problem of robust stabilization in the gap metric as in the work of Georgiou and Smith [1]. In this paper, we formulate a state-feedback version of the problem of finding maximally robust controllers for a plant with normalized right coprime factor uncertainty. (See the next section for details.) We show that a maximally robust state-feedback controller for stabilization of a plant subject to uncertainty in its normalized right coprime factors is given by a linear quadratic regulator $(L Q R)$ gain. Thus, this paper shows that the LQR gain enjoys an additional robustness property. This result is really an observation as it is quite easy to prove; however, it appears to not have been noticed in the existing literature.

The notation is quite standard. The transpose of a matrix $A$ is denoted by $A^{\prime}$ and $G^{*}(s):=G^{\prime}(-s)$. The maximum eigenvalue of a real symmetric matrix $A$ is denoted by $\lambda_{\max }(A)$. The normed linear space of proper stable real rational functions with the $\mathscr{H}_{\infty}$ norm is denoted by $\mathscr{R} \mathscr{H}_{\infty}$. A transfer matrix in terms of state-space data is denoted by

$\left[\begin{array}{l|l}A & B \\ \hline C & D\end{array}\right]:=C(s I-A)^{-1} B+D$.

\section{Main result}

Consider the (nominal) system $\Sigma$ :

$$
\begin{aligned}
& \dot{x}=A x+B u, \\
& v=C x+D u, \\
& y=x .
\end{aligned}
$$


Note that we are assuming that the measured output for the nominal plant model is $y=x$ - the state of the nominal model. After we introduce plant uncertainty, the measured output $y=x$ will be affected by the uncertainty as will be discussed later.

Next we will describe how the uncertainty enters this nominal model by introducing perturbations in a normalized coprime factorization of transfer function $G_{v u}:=D+C(s I-A)^{-1} B$. For simplicity, we will assume that the triple $(A, B, C)$ is minimal. Consider the algebraic Riccati equation (ARE)

$$
\begin{gathered}
\left(A-B S^{-1} D^{\prime} C\right)^{\prime} X+X\left(A-B S^{-1} D^{\prime} C\right) \\
-X B S^{-1} B^{\prime} X+C^{\prime} R^{-1} C=0
\end{gathered}
$$

where $S:=I+D^{\prime} D, R:=I+D D^{\prime}$. Since $(A, B, C)$ is minimal, it follows that there exists a unique symmetric positive definite solution $X$ to (2.4). The following state-space construction for the normalized right coprime factorizations was given by Vidyasagar [6].

Lemma 2.1. Consider the system $\Sigma$, and assume $(A, B, C)$ is minimal. Let $X$ be the (unique) symmetric positive definite solution to (2.4), and $F:=-S^{-1}\left(D^{\prime} C+B^{\prime} X\right)$. Define

$$
\begin{aligned}
& M:=\left[\begin{array}{c|c}
A+B F & B S^{-1 / 2} \\
\hline F & S^{-1 / 2}
\end{array}\right], \\
& N=\left[\begin{array}{c|c}
A+B F & B S^{-1 / 2} \\
\hline C+D F & D S^{-1 / 2}
\end{array}\right] .
\end{aligned}
$$

Then $G_{v u}=N M^{-1}$ is a normalized right coprime factorization of the transfer function matrix $G_{v u}$, i.e. $N, M$ are in $\mathscr{R}_{\infty} \mathscr{H}_{\infty}$, they are right coprime, and $M^{*} M+N^{*} N=I$.

The normalized coprime factors $M, N$ are unique modulo multiplication on the right by a real constant orthogonal matrix. This nonuniqueness has no effect on the results to follow.

As in Glover and McFarlane [2], let us consider perturbations of $M, N$, which are normalized coprime factors of $G_{v u}$. We will first describe this in terms of the state-space realization of the nominal system $\Sigma$. Consider the perturbed system $\Sigma_{\mathrm{p}}$ :

$$
\begin{aligned}
& \dot{x}=A x+\left[\begin{array}{ll}
-B & 0
\end{array}\right]\left[\begin{array}{l}
w_{1} \\
w_{2}
\end{array}\right]+B u, \\
& z=-S^{1 / 2} F x+\left[\begin{array}{ll}
-S^{1 / 2} & 0
\end{array}\right]\left[\begin{array}{l}
w_{1} \\
w_{2}
\end{array}\right]+S^{1 / 2} u,
\end{aligned}
$$

$\left[\begin{array}{l}w_{1} \\ w_{2}\end{array}\right]=\left[\begin{array}{l}\Delta_{M} \\ \Delta_{N}\end{array}\right] z$

$v=C x+\left[\begin{array}{ll}-D & I\end{array}\right]\left[\begin{array}{l}w_{1} \\ w_{2}\end{array}\right]+D u$,

$y=x$.

Here $\Delta_{M}, \Delta_{N}$ are stable unknown transfer functions which represent the uncertainty in the nominal plant model. An easy calculation shows that for the perturbed system,

$G_{v u}=\left(N+\Delta_{N}\right)\left(M+\Delta_{M}\right)^{-1}$.

A block diagram showing this interconnection is shown in Figure 1. Note that for the above open loop uncertain system to be proper (and wellposed), $\left(I+M^{-1} \Delta_{M}\right)$ should be invertible and the inverse should be proper.

The state-feedback assumption thus amounts to assuming that the state of the above perturbed plant is available for feedback. As we have seen above, it represents the state of the nominal system plus the effect of the plant perturbations. Also note that only $w_{1}$ affects $x$ but not $w_{2}$. This is simply a consequence of the way normalized right coprime factor perturbations enter the system.

A controller $K$ is said to be admissible if it internally stabilizes the nominal plant $\Sigma$. For a given

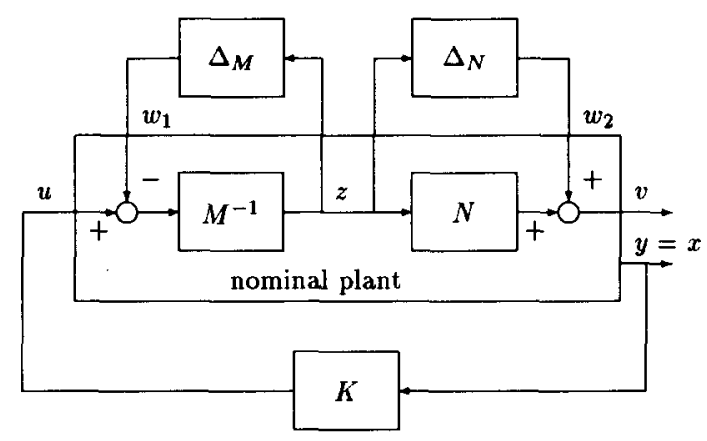

Fig. 1. Right coprime factors perturbations. 
(possibly dynamic) admissible controller $K$, the robust stability margin $\varepsilon(K)$ is defined as follows:

$\varepsilon(K):=\sup \{\varepsilon:$ the closed loop system is well-posed and asymptotically stable for all $\Delta_{M}, \Delta_{N} \in \mathscr{R} \mathscr{H}_{\infty}$ with $\left.\left\|\left[\begin{array}{l}\Delta_{N} \\ \Delta_{M}\end{array}\right]\right\|_{\infty} \leq \varepsilon\right\}$.

The robust stabilization objective is to find a controller $K$ which maximizes $\varepsilon(K)$. Note that $\Delta_{N}$ plays no role in robust stability in this state-feedback case. The next theorem gives a maximally robust state-feedback controller.

Theorem 2.2. Consider the system $\Sigma_{\mathrm{p}}$. Then a statefeedback controller which maximizes the stability margin $\varepsilon(K)$ is the $L Q R$ gain $K=F:=$ $-S^{-1}\left(D^{\prime} C+B^{\prime} X\right)$ and the maximum stability margin $\varepsilon_{\max }$ is $\left(1+\lambda_{\max }\left(D^{\prime} D\right)\right)^{-1 / 2}$.

Proof. Let $K$ be an admissible controller. Let $T_{z w}$ denote the closed loop transfer function from $w$ to $z$. It is easy to see that

$T_{z w}=S^{1 / 2}[K(s)-F] T_{x w}+\left[\begin{array}{ll}-S^{1 / 2} & 0\end{array}\right]$.

As $T_{x w}$ is strictly proper, it follows that

$$
\left\|T_{z w}\right\|_{\infty} \geq\left\|T_{z w}(\infty)\right\|=\left(1+\lambda_{\max }\left(D^{\prime} D\right)\right)^{1 / 2} .
$$

Now by the small gain theorem, we can find a stable rational transfer function $\Delta$ such that $\|\Delta\|_{\infty} \leq\left(1+\lambda_{\max }\left(D^{\prime} D\right)\right)^{-1 / 2}$ and the closed loop system is either unstable or not well-posed. It follows that for any admissible controller,

$\varepsilon(K) \leq\left(1+\lambda_{\max }\left(D^{\prime} D\right)\right)^{-1 / 2}$.

Now consider the state-feedback control law

$u=F x=-S^{-1}\left(D^{\prime} C+B^{\prime} X\right) x$.

This is an admissible controller. The closed loop transfer function (with $\Delta_{M}=0, \Delta_{N}=0$ ) from $w$ to $z$ is given by $T_{z w}=\left[-S^{1 / 2} 0\right]$ and

$\left\|T_{z w}\right\|_{\infty}=\left\|S^{1 / 2}\right\|=\left(1+\lambda_{\max }\left(D^{\prime} D\right)\right)^{1 / 2}$.

Now the perturbed system is well-posed, and by the small gain theorem the perturbed closed loop system is internally stable provided

$\left\|\left[\begin{array}{c}\Delta_{N} \\ \Delta_{M}\end{array}\right]\right\|_{\infty}<\left(1+\lambda_{\max }\left(D^{\prime} D\right)\right)^{-1 / 2}$.
Therefore,

$\varepsilon(K=F)=\left(1+\lambda_{\max }\left(D^{\prime} D\right)\right)^{-1 / 2}$.

Remark 1. The controller $u=F x=-S^{-1}\left(D^{\prime} C+\right.$ $\left.B^{\prime} X\right) x$ given by $\operatorname{ARE}(2.4)$ is the solution to the following standard LQR problem. Consider the system $\Sigma$ :

$\dot{x}=A x+B u, \quad v=C x+D u$.

Minimize the cost functional:

$\int_{0}^{\infty}\left(v^{\prime} v+u^{\prime} u\right) \mathrm{d} t$

It should also be noted that while we are considering state-feedback controllers, the output matrices which are used in setting up the LQR problem are precisely the same as those used in defining the uncertainty structure.

A significant simplification is obtained in the case of strictly proper plants, i.e. $D=0$. In this case, the maximum stability margin is one, the ARE simplifies to the familiar LQR algebraic Riccati equation,

$A^{\prime} X+X A-X B B^{\prime} X+C^{\prime} C=0$,

and a maximally robust state-feedback controller is $K=F=-B^{\prime} X$.

Remark 2. It is not difficult to see for any $\varepsilon<\left(1+\lambda_{\max }\left(D^{\prime} D\right)\right)^{-1 / 2}$, and a stable perturbation $\Delta$ such that $\|\Delta\|_{\infty} \leq \varepsilon$, the corresponding perturbed system $\Sigma_{\mathrm{p}}$ is proper (and well-posed). On the other hand, suppose that $\varepsilon \geq\left(1+\lambda_{\max }\left(D^{\prime} D\right)\right)^{-1 / 2}$. It is easy to see that we can find a real matrix $\Delta_{M}$ such that $\left(I+S^{-1 / 2} \Delta_{M}\right)$ is singular, while

$$
\left\|\Delta_{M}\right\|_{\infty}=\left\|S^{1 / 2}\right\|^{-1}=\left(1+\lambda_{\max }\left(D^{\prime} D\right)\right)^{-1 / 2} \leq \varepsilon .
$$

With such a choice of $\Delta_{M}$ and $\Delta_{N}=0$, the perturbed system $\Sigma_{\mathrm{p}}$ is not proper. Thus, the boundary of stabilizability coincides with the possible loss of properness of the perturbed system in the statefeedback case.

It is interesting to note that in the output feedback case, the situation is quite different. In this case, there may exist coprime factor perturbations whose norm is less than the radius of stabilizability but the open loop perturbed system $\Sigma_{p}$ is not proper. It should be noted that the closed loop system is certainly well-posed and proper for all perturbations within the guaranteed robust 
stability radius. Such examples may be obtained by taking a nominal SISO plant with a very large $D$ term. The reason for this situation is that the properness of $\Sigma_{p}$ is not imposed as a constraint on the family of perturbed plants. Rather, only the well-posedness of the closed loop system consisting of the perturbed plant transfer function $G_{v u}$ (which may be improper) and the controller transfer function $K$ is required.

\section{References}

[1] T.T. Georgiou and M.C. Smith, Optimal robustness in the gap metric, IEEE Trans. Automat. Control 35 (1990) $673-686$.
[2] K. Glover and D.C. McFarlane, Robust stabilization of normalized coprime factor plant descriptions with $\mathscr{H}_{\infty}$ bounded uncertainty, IEEE Trans. Automat. Control 34 (1989) 821-830.

[3] R.E. Kalman, When is a linear control system optimal? Trans. ASME, Ser. D (J. Basic Engr.) 86 (1964) 51-60.

[4] D.C. McFarlane and K. Glover, Robust controller design using normalized coprime factor plant descriptions, Lecture Notes in Control and Information Sciences, Vol. 138 (Springer, Berlin, 1990).

[5] M.G. Safonov and M. Athans, Gain and phase margin for multiloop LQG regulators, IEEE Trans. Automat. Control 22 (1977) 173-179.

[6] M. Vidyasagar, Normalized coprime factorizations for nonstrictly proper systems, IEEE Trans. Automat. Control 33(3) (1988) 300-301.

[7] M. Vidyasagar and H. Kimura, Robust controllers for uncertain linear multivariable systems, Automatica, 22(1) (1986) 85-94. 